\title{
A Revised Version of the ATLAS Tile Calorimeter Link Daughterboard for the HL-LHC
}

\author{
Eduardo Valdes Santurio ${ }^{\circledR}$, Samuel Silverstein ${ }^{\circledR}$, Christian Bohm ${ }^{\circledR}$, Life Senior Member, IEEE, \\ Katherine Dunne ${ }^{\circledR}$, Suhyun Lee ${ }^{\circledR}$, and Holger Motzkau
}

\begin{abstract}
The ATLAS Tile Calorimeter (TileCal) readout link and control daughterboard (DB) is the central on-detector hub of the new TileCal electronics upgrade for the high-luminosity large hadron collider (HL-LHC). The DB, which has undergone gradual redesigns during development, provides the connection between the on- and off-detector electronics via bidirectional fiber-optic links. Two CERN-developed, radiation hard GBTx application specified integrated circuits (ASICs) receive LHC timing signals and configuration commands through $4.8-\mathrm{Gb} / \mathrm{s}$ downlinks, which are in turn propagated to the front end through Xilinx Kintex Ultrascale field-programmable gate arrays (FPGAs). The Kintex FPGAs also continuously perform real-time readout and transmission of digitized photomultiplier (PMT) samples, detector control system (DCS) signals, and monitoring data through redundant pairs of 9.6-Gb/s uplinks. The DB design aims at minimizing single points of failure and improving the performance and reliability of the board. Apart from the GBTx devices, the DB design relies on radiation-qualified commercial off-the-shelf (COTS) components. Mitigation of radiationinduced single-event upsets (SEUs) in the FPGAs is performed by a combination of the Xilinx soft error mitigation (SEM) controller and triple-mode redundancy (TMR) schemes in the FPGA firmware. Data integrity is protected through forward error correction (FEC) in the downlinks and cyclic redundancy check (CRC) error verification in the redundant uplinks. This article presents the latest revision of the DB (version 6), a redesign that addresses single-event latch-up (SEL) behavior observed in the Kintex Ultrascale+ FPGAs used in the previous revision, and features a more robust power circuitry combined with an improved current monitoring scheme, enhanced performance of the analog-to-digital converter (ADC) read-out, and improved timing performance.
\end{abstract}

Index Terms-Link hub, nonionizing energy losses (NIELs), single-event effects (SEEs), single-event latch-up, triple-mode redundancy (TMR).

\section{INTRODUCTION}

$\mathbf{T}$ HE high-luminosity large hadron collider (HL-LHC) program involves a series of upgrades to the accelerator aimed at increasing the instantaneous luminosity to at least five times the original design value. This upgrade will allow the LHC to increase the volume of collision data provided

Manuscript received June 14, 2021; revised July 26, 2021 and July 30, 2021; accepted August 5, 2021. Date of publication August 9, 2021; date of current version September 16, 2021. This work was supported in part by Stockholm University, in part by CERN, and in part by the Swedish Research Council (Vetenskapsrådet).

The authors are with the Department of Physics (Fysikum), Stockholm University, 11421 Stockholm, Sweden (e-mail: eduardo.valdes@fysik.su.se).

Color versions of one or more figures in this article are available at https://doi.org/10.1109/TNS.2021.3103408.

Digital Object Identifier 10.1109/TNS.2021.3103408

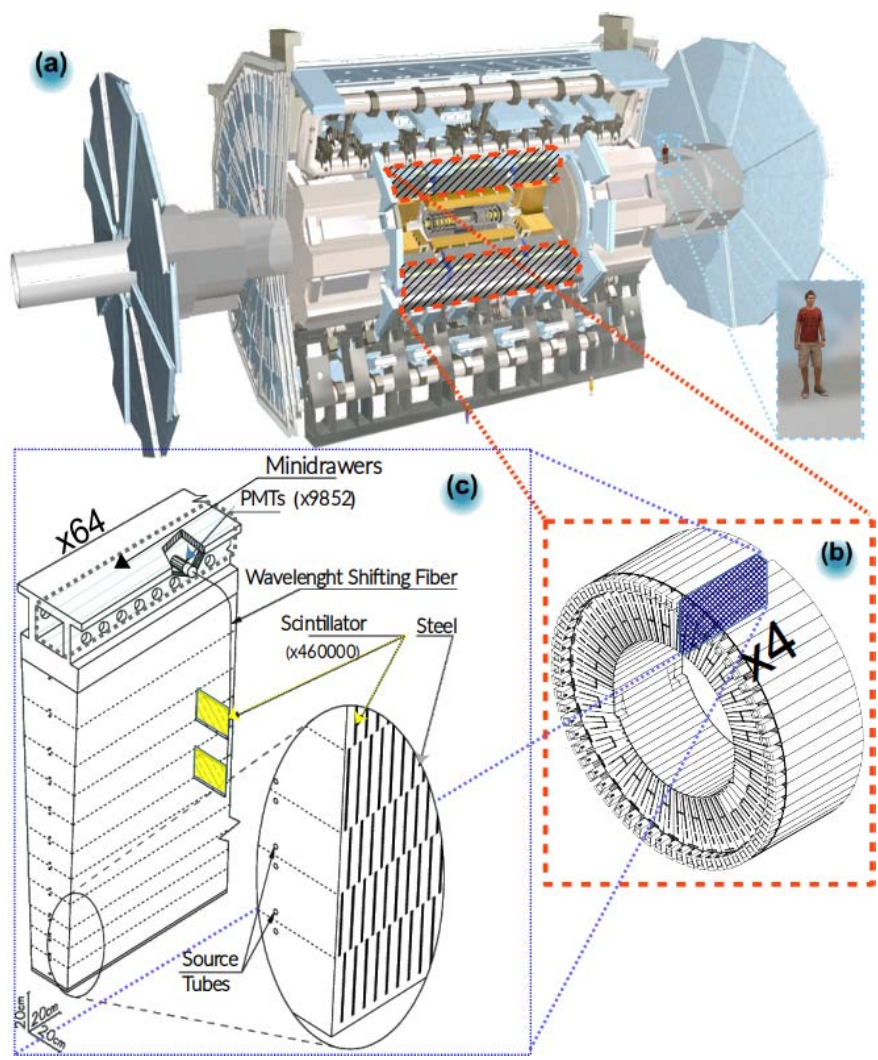

Fig. 1. (a) ATLAS detector. (b) TileCal barrel. (c) Depiction of a TileCal wedge-shaped module [1].

to the experiments by an order of magnitude. The higher LHC luminosity will come with a corresponding increase of radiation exposure to the detectors and the on-detector read-out electronics systems, as well as increased rates of low-momentum collision backgrounds (pileup). To prepare for this, extensive R\&D work is ongoing to ensure that the read-out electronics of the upgraded ATLAS detector [1] are capable of meeting the new HL-LHC requirements.

\section{ATLAS TILE CALORIMETER}

The ATLAS Hadronic Tile Calorimeter (TileCal) is a sampling calorimeter with plastic scintillator tiles and steel plates as active and absorber materials, respectively. TileCal is formed by three cylindrical barrel sections that altogether correspond to four partitions [Fig. 1(b)]. Each partition comprises 64 wedge-shaped modules [Fig. 1(c)] that are divided into cells 
that form pseudo-projective towers aimed at the interaction point. The light produced by the scintillating tiles in each cell is collected by wavelength-shifting fibers and read out by a pair of photomultipliers (PMTs).

For the HL-LHC upgrade, all of the on- and off-detector electronics will be replaced with new systems designed to provide continuous digital read-out of all calorimeter cells, with lower electronic noise and better timing stability. The trigger tower sums sent to the Level-1 trigger will be performed digitally from individual PMT data, making them less sensitive to out-of-time pileup and improving energy resolution [2]. The upgraded on-detector electronics will be tested and qualified for total ionizing dose (TID), nonionizing energy loss (NIEL), and single-event effects (SEEs), to assure reliability through the full HL-LHC running period.

\section{TILECAL UPGRAde FOR THE HL-LHC}

The HL-LHC TileCal on-detector electronics are mechanically partitioned into 896 independent modules, so-called MiniDrawers [MD, Fig. 2(a)]. Three or four MDs are installed within the rear beams of each wedge-shaped module, where bundles of wavelength-shifting fibers corresponding to one side of each cell are brought to pairs of PMTs in the MD. Each MD consists of an aluminum mechanical structure that can house up to 12 PMTs. Each PMT is equipped with front-end boards called front-end boards for the new infrastructure with calibration and signal shaping (FENICs), which shape, condition, and amplify the analog PMT signals. The FENICS boards produce low- and high-gain analog output signals with a 1:40 ratio and send them to a mainboard (MB) for digitization [see Fig. 2(b)].

The daughterboard (DB) is mounted onto the MB [see Fig. 2(b) and (c)]. It continuously reads, formats, and transfers PMT data from the MB to the off-detector systems while receiving and distributing LHC-synchronized timing, control signals, and configuration commands to the front-end electronics. In the off-detector systems, Tile PreProcessors (TilePPr) continuously receive and store PMT digitized data in pipelines while awaiting a trigger decision. They also produce and transmit tower sums to the Level-0 trigger system (L0) and read out triggered event data to the Front-End Link eXchange (FELIX) system [8] via the Trigger and Data Acquisition interface (TDAQi).

\section{DB REVISION 5}

The version $6 \mathrm{DB}$ presented in this document is a significant redesign of the previous revision 5 board (DB5, Fig. 3), Several important features of the redesign are motivated by experience from DB5, so we present it here too for context. The DB design follows redundant scheme, where each half of the board is independently powered and can run standalone if needed. A CERN developed, radiation-hard timing and transceiver IC (GBTx) [9] on each of the DB halves (A and B) receives two independent sets of clock signals, commands, and configuration interfaces through dedicated fiber-optic downlinks (through separate SFP+ modules) and distributes one set to each half of the DB. This allows both halves of the DB to continue to run nominally if one downlink fails. Each DB half provides redundant uplinks to the off-detector systems, by means of two separate SFP+ modules. Each of the small form-factor pluggable transceiver (SFP) modules on each half sends identical data. Therefore, failure of any one of the four $\mathrm{SFP}+$ modules will not compromise data taking of any of the halves.

The GBTx ASICs each recover two LHC-synchronous 40$\mathrm{MHz}$ clocks to drive the real-time functionality of the two large field-programmable gate arrays (FPGAs), two pairs of phase-configurable $40-\mathrm{MHz}$ clocks to drive the analog to digital converter (ADC) clocks in the different MB quadrants, and two $160-\mathrm{MHz}$ synchronous clocks for the multigigabit transceiver (MGT) blocks that drive the 9.6-Gb/s uplinks. In addition, the GBTx ASICs receive and decode data words from the off-detector systems, including checksums for forward error correction (FEC) to provide configuration and control commands, reset signals, and access to the FPGA configuration ports (JTAG) to both FPGAs through dedicated ports (E-Ports). The aforementioned JTAG interfaces allow write-only reconfiguration of the Kintex Ultrascale+ $(\mathrm{KU}+)$ FPGAs and their attached flash memories through the off-detector electronics. The FPGA firmware implements triple-mode redundancy (TMR), and readout of the MB data is transmitted on redundant links using GBT-cyclic redundancy check (CRC) protected words.

Earlier revisions before DB5 were based on Kintex-7 (K7) FPGAs with GTX MGT blocks. These were found to be incompatible with LHC timing [3] because the GTX MGTs are not specified for the interval from 9.4 to $9.8 \mathrm{~Gb} / \mathrm{s}$, putting the bitrate of $9.6-\mathrm{Gb} / \mathrm{s}$ rate outside of the specifications. Furthermore, the quad-phase-locked-loop (QPLL) modules of the GTX MGTs were not able to generate the $9.6-\mathrm{Gb} / \mathrm{s}$ bitrates with the GBTx clocks available (40, 80, 160, and $320 \mathrm{MHz}$ ). Therefore, the DB5 design was migrated to the new KU+ FPGAs with GTY MGT technology that supports $9.6-\mathrm{Gb} / \mathrm{s}$ link speeds driven directly by GBTx reference clocks. The $\mathrm{KU}+$ FPGAs were chosen over the Kintex Ultrascale (KU) architecture because their smaller feature size (16-nm FinFET versus 20 -nm planar) was expected to provide better radiation tolerance and lower rates of single-event upsets (SEUs) [5].

The DB5 provided backward compatibility with earlier revisions, including the 400-pin FMC docking connector to the $\mathrm{MB}$, and cable interfaces with the Cesium calibration system connector (Cs Interface) and the on-detector highvoltage interface (HV-OPTO). A commercial connector with custom pin assignment was routed to the Xilinx analog-todigital converter (xADC) of each FPGA for extra sensor signal monitoring, and DS20CM00 digital ID serial chips were added for in situ digital identification of the MD.

\section{TID, NIEL, AND SEE RADIATION TESTS ON THE DB 5}

The DB5 was successfully tested under power for TID in March 2018 in 9- and 12-MeV electron beams at a Varian Clinac facility, Karolinska Hospital, Stockholm. A total of $20 \mathrm{kRad}$ was delivered in six runs, corresponding to ten years of HL-LHC exposure plus large safety factors. The test protocol followed the simulated radiation doses reported in the ATLAS Tile Calorimeter Phase-II Upgrade Technical 


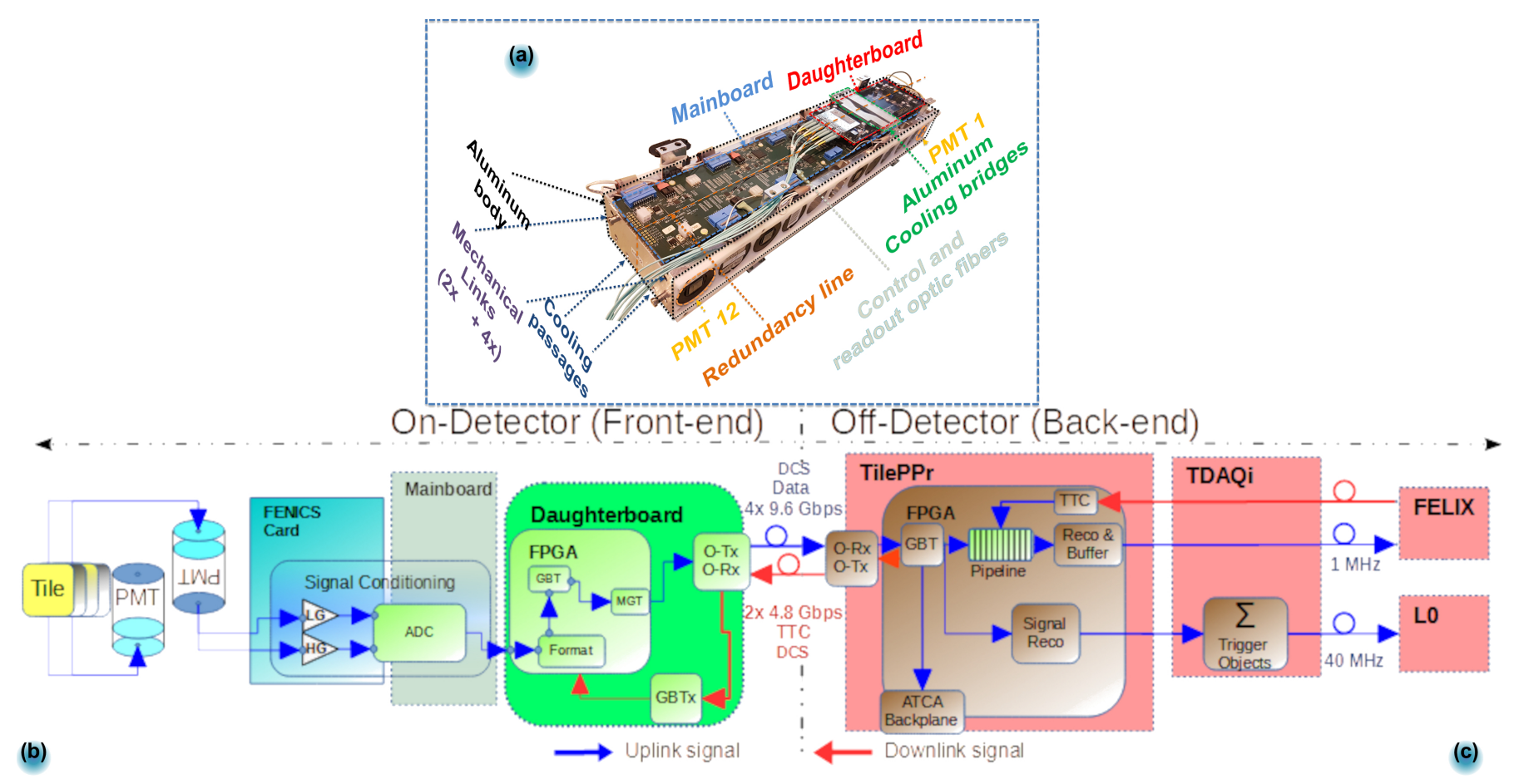

Fig. 2. TileCal HL-LHC upgrade read-out system. (a) TileCal Phase-II upgrade MD. (b) On-detector electronics block diagram. (c) Off-detector electronics block diagram.

Design Report in [2]. However, subsequent simulations with updated geometry and new guidelines have produced new dose estimates that motivate a new round of TID tests. The new simulated values were taken into consideration for the NIEL and SEE tests.

The NIEL test was performed in May 2018 with a $52-\mathrm{MeV}$ proton beam at the AIC-144 cyclotron at The Henryk Niewodniczański Institute of Nuclear Physics (IFJ-PAN), Krakow. To evenly irradiate a sufficiently large area of the DB to include every type of component, a double-Gaussian distribution was produced with two beam exposures with a 148-mm offset, for a homogeneity of $\pm 10 \%$ over a $100 \mathrm{~mm} \times 200 \mathrm{~mm}$ region that was verified with film dosimetry. The delivered fluence of $5.00 \times 10^{12}$ protons $\times \mathrm{cm}^{-2}$ corresponded to $9.00 \times 10^{12}$ neutrons $\times \mathrm{cm}^{-2}$ (1-MeV equivalent neutrons). In addition to the DB5, the test included two different SFP+ devices, AVAGO AFBR-709SMZ and CORETEK CT000NPP-SB1L-D. The microcontroller present on the irradiated AVAGO SFP+ completely lost functionality, with no power consumption or signal detected when powered. On the other hand, the irradiated CORETEK SFP+ showed no detectable signal degradation. The GBTx ASICs and the FPGAs on the DB5 were unfortunately found to be severely damaged, with electrical shorts on the various supply voltages, almost certainly due to the high ionizing dose $768.2 \mathrm{kRad}(\mathrm{Si})$ from the proton beam over a relatively small time period of $84 \mathrm{~min}$. The NIEL test was thus considered inconclusive, and new tests at a neutron beam facility are planned.

The SEE campaign in November 2018 included tests in two proton beamlines at IFJ-PAN, Krakow (Fig. 4). The first test used a $58-\mathrm{MeV}$ proton beam at the AIC facility with 4-cm-diameter field size to irradiate one FPGA at a time, with remote JTAG monitoring of the internal soft error mitigation (SEM) to count SEU rates in the configuration memory. A total of 16 runs were performed, for a total fluence of $2.5 \times 10^{11}$ protons $\times \mathrm{cm}^{-2}$. Of these, 11 runs corresponding to $2.02 \times 10^{10}$ protons $\times \mathrm{cm}^{-2}$ yielded usable SEU data, with 5334 SEUs observed for a rate of $2.8 \times 10^{-8}$ per KU3P device, or $2.3 \times 10^{-16}$ per bit. Data for the other five $58-\mathrm{MeV}$ runs were lost when communications were disrupted by unexpected latch-up events, for a singleevent latch-up (SEL) rate of $2.0 \times 10^{-11}$ per device.

The second SEE test at IFJ-PAN was performed in a therapeutic scanning proton beam at the Cyclotron Center Bronowice (CCB) facility using $226-\mathrm{MeV}$ protons. A total fluence of $1.44 \times 10^{11}$ protons $\times \mathrm{cm}^{-2}$ was delivered to the DB, while currents were remotely monitored. Power was cycled after each detected SEL to prevent damage to the FPGA. A total of 31 SELs were observed and characterized (Figs. 5 and 6), for a rate of $2.15 \times 10^{-10}$ per device (Fig. 7).

We had not expected to see latch-up in the KU+ FPGAs since the previous seven-series and Ultrascale families do not have this problem and the low core logic voltage (VCORE) $(0.85 \mathrm{~V})$ is well below the $1.3-\mathrm{V}$ threshold required to forward-bias a latent thyristor structure (PNPN) with two $0.65-\mathrm{V}$ junctions in series. While the exact mechanism is not clearly understood from the data, it can be seen that each SEL event is associated with a slow and steady current rise in one of the I/O reference voltages $(1.8,2.5$, and $3.3 \mathrm{~V})$. This is consistent with contemporary results for heavy-ion SEE tests [4], with the vast majority of SEL-like errors seen in the 1.8-V VCCAUX powering the I/O banks of the tested device. 


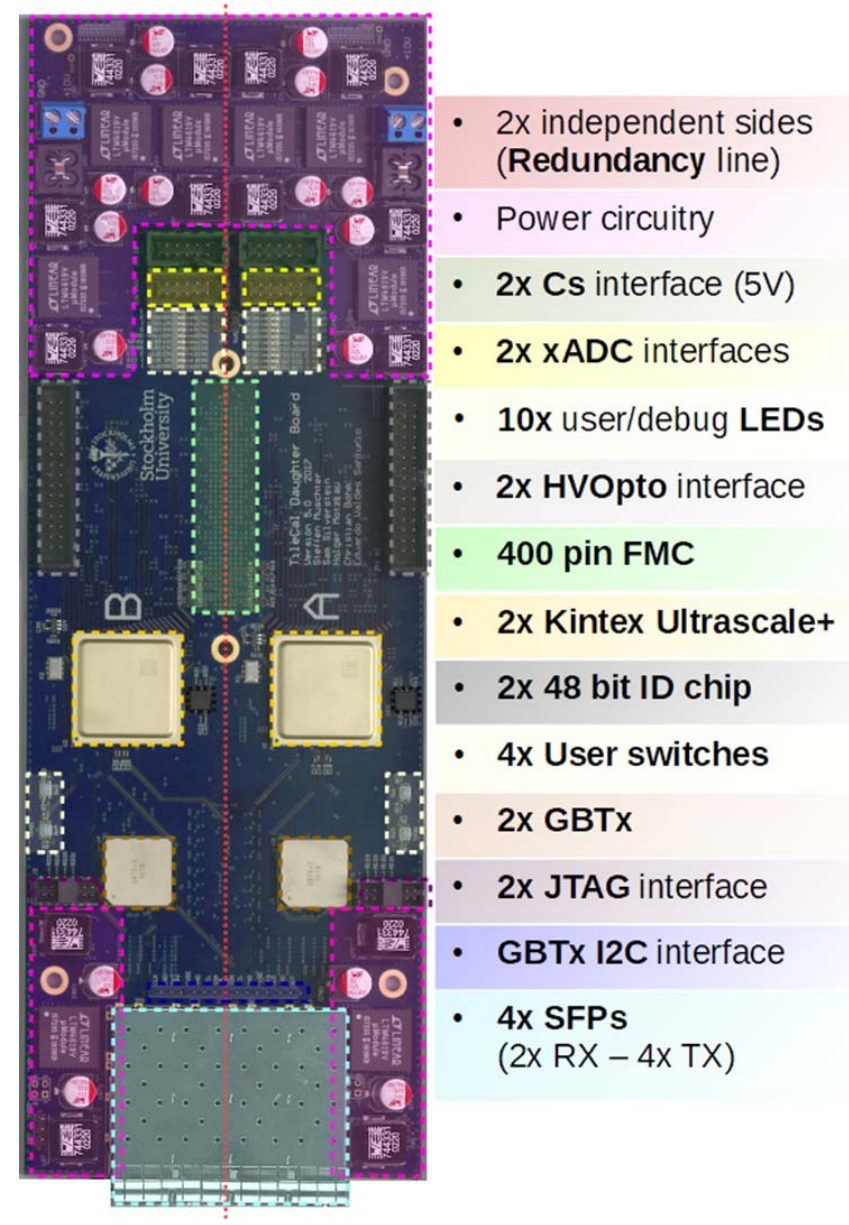

Fig. 3. DB revision 5 .

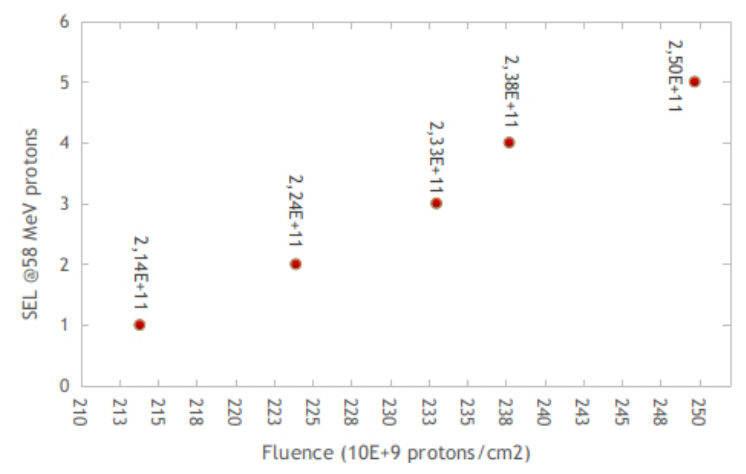

Fig. 4. Detected single-event latch-ups of DB version 5 during the test with $58-\mathrm{MeV}$ protons.

Although the KU+ FPGAs under test were exposed to overcurrents for periods up to around $60 \mathrm{~s}$, the device functionalities were always recovered after a quick power cycle. In addition, no detectable damage to the FPGAs was seen in long test runs performed well after the irradiation test. However, the disruption to data taking from the measured SEL rates is not acceptable for HL-LHC running. This motivates a new DB design based on KU FPGAs, which are more resistant to latch-up.

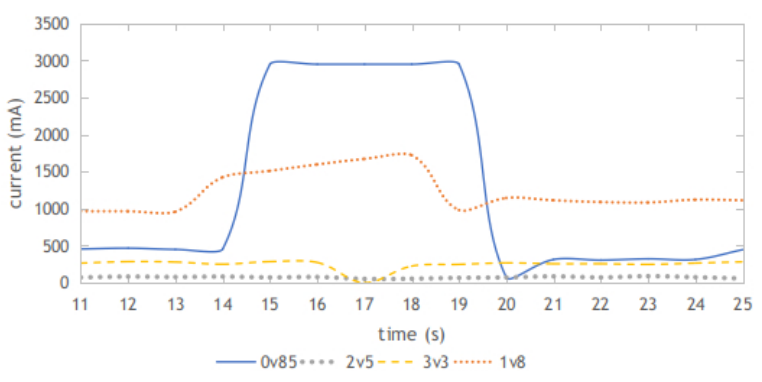

Fig. 5. Single-event latch-up overcurrent seen for half A of DB 5 during the SEU test performed with protons at $226 \mathrm{MeV}$. A quick power cycle was performed $5 \mathrm{~s}$ after the overcurrent appeared in the VCORE voltage.

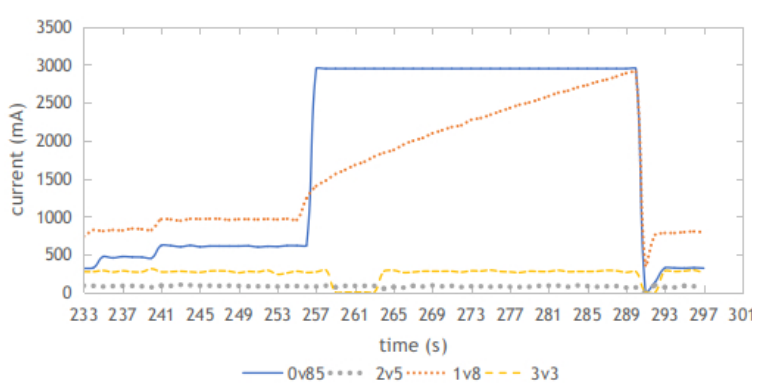

Fig. 6. Single-event latch-up overcurrent seen for half A of DB 5 during the SEU test performed with protons at $226 \mathrm{MeV}$. A quick power cycle was performed $40 \mathrm{~s}$ after the overcurrent appeared in the VCORE voltage to verify that the $1.8 \mathrm{~V}$ reached the $3-\mathrm{A}$ maximum current measurable by the setup.

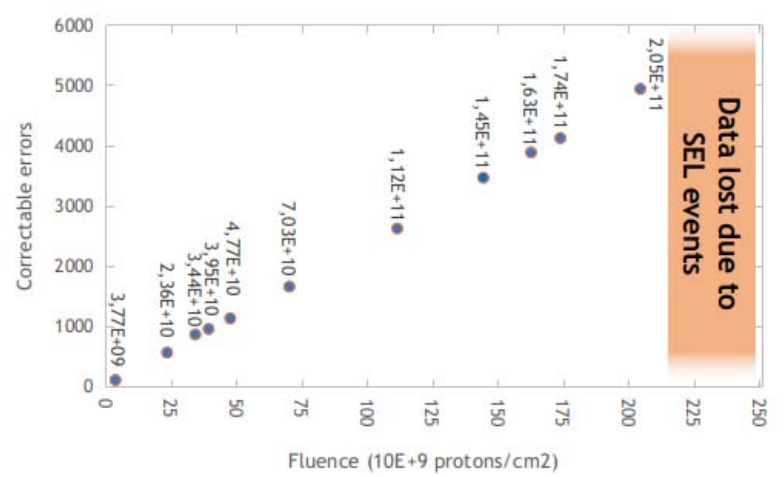

Fig. 7. SEUs for the KU+ FPGA in DB 5 performed with protons at $58 \mathrm{MeV}$. Each point represents the number of errors corrected by the Xilinx SEM before an uncorrectable error appeared. The firmware was reloaded from the flash memories after an uncorrectable error event. Data corresponding to a fluence of approximately 0.45 protons/cm was lost due to the SEL overcurrents interfering with the data-taking process.

Of the 5334 SEUs counted, 11 were classified as "uncorrectable" by the SEM. At low SEU rates, these are almost always due to a corrupted error-correcting code (ECC) detected by the CRC frames of the SEM. While these prevent the SEM from correcting further errors, they do not in fact affect the actual function of the FPGA configuration. Although uncertainties are high, if we assume an average fluence at HL-LHC corresponding to $1.4 \times 10^{-10} \mathrm{~cm}^{-2} /$ year, we would expect on the order of one correctable error/FPGA/day and three uncorrectable errors/FPGA/year. Correctable errors will be quickly corrected by the SEM, whereas uncorrectable errors 


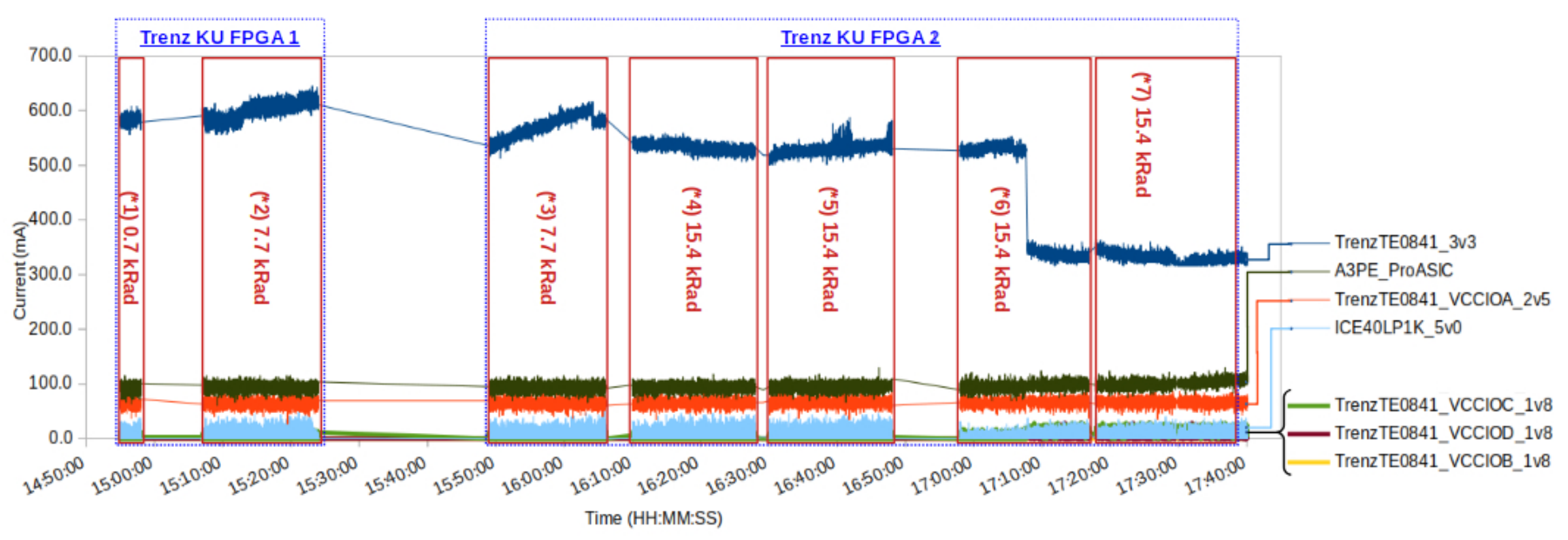

Fig. 8. Current monitoring data corresponding to SEL tests with protons at $226 \mathrm{MeV}$ performed on two Trenz TE0841 micromodules (marked as Trenz KU FPGA 1 and Trenz KU FPGA 2), a Microsemi A3PE starter kit, and a Lattice ICEBLINK LP1K evaluation board. The irradiation took place over seven runs, each signalized in the red boxes with $(* x)$ followed the corresponding deposited TID dose. Note that values for the measured currents for TrenzTE0841_VCCIOB, TrenzTE0841_VCCIOC, and TrenzTE0841_VCCIOD are quite close to the current values for the ICE40LP1K_5V0. Therefore, the aforementioned channels are not visible in the plot.

can be cleared by reconfiguring the affected FPGA at the next convenience.

SEU-induced transient errors or corrupted configuration bits in the parts of the firmware that are implemented in the fabric of the FPGAs are mitigated by the use of triple-mode redundancy. This is implemented using triplicated copies of control registers and real-time data path stages, with voting circuits to suppress single-point errors over the short period before they are corrected by the SEM. We, therefore, do not expect SEUs to have a meaningful effect on data taking at HL-LHC.

\section{REDESIGNING THE DB}

The DB5 was a significant step forward from earlier DB revisions, including extensive firmware development and testing with different hardware prototypes for the HL-LHC upgrade. However, experience with DB5 exposed a number of design flaws that needed to be addressed in the new design.

The DB5 power-on sequence was implemented with parallel $R C$ circuits connected to the "run" pins of the various LTM4619 dc-dc converters providing the different voltages. However, these can be sensitive to aging or power ramping and do not disable a later voltage in the sequence from powering on if one of the earlier sources fails. This can potentially cause damage to the FPGAs. A new power scheme was developed to provide fail-safe power sequencing while also detecting and mitigating unexpected overcurrents.

The remote JTAG interfaces between the GBTxs and the FPGAs proved to be unstable when the GBTx downlink was lost due to scenarios such as optic link failures or problems on the TilePPr transmission. This was found to be related to the instability of the GBTx RX_RDY signal and the DATA_VALID GBTx signals that were used to enable the buffers driving the JTAG chains. To mitigate the effects of the instabilities, the remote JTAG interface was migrated to a small FPGA with more sophisticated functionality designed to prevent a GBTx with a lost link from accidentally activating the interface.

Full performance of the $9.6-\mathrm{Gb} / \mathrm{s}$ uplinks was not achieved because of jitter present in the phase-adjustable (deskew) GBTx clocks used to drive the GTY MGTs. To solve this problem, the reference clocks were routed to GBTx E-Link clock outputs. The GBTx E-Link outputs do not provide deskew capabilities but have lower jitter than the clock outputs provided by the GBTx phase shifter.

DB5 tests of ADC readout with various $\mathrm{MB}$ revisions showed reduced performance due to nonoptimal routing of the ADC clocks and data signals to the FPGA I/O banks at the board level. This issue needed to be addressed by optimizing the routing to assure that there are no timing violations during the implementation of the ADC readout firmware block because of the physical routing at the board level. In addition, the deskew clock distribution scheme to the different MB quadrants needed to be redesigned.

As discussed above, the SEL rates seen in DB5 could be eliminated by migrating back to Ultrascale FPGAs with a 20-nm planar technology (TSMC), where SELs have not been observed [6]. One disadvantage of this migration from the $\mathrm{KU}+$ architecture to the $\mathrm{KU}$ architecture is that the SEU rate is expected to increase by a factor of approximately 16 [5]. This corresponds to an average soft error rate of less than one per hour per FPGA, which is still well within our mitigation capabilities using SEM and TMR.

\section{SEL TESTS FOR THE DB 6 FPGAs}

Our chosen KU FPGA contains GTH MGTs that are compatible with the 160-MHz reference clocks provided by the GBTx and is expected to have manageable SEU rates. Two small FPGAs were chosen as candidates for the new JTAG interface: a Microsemi ProASIC3 flash-based FPGA and a Lattice ICE40LP nonvolatile configuration memory-based FPGA. 


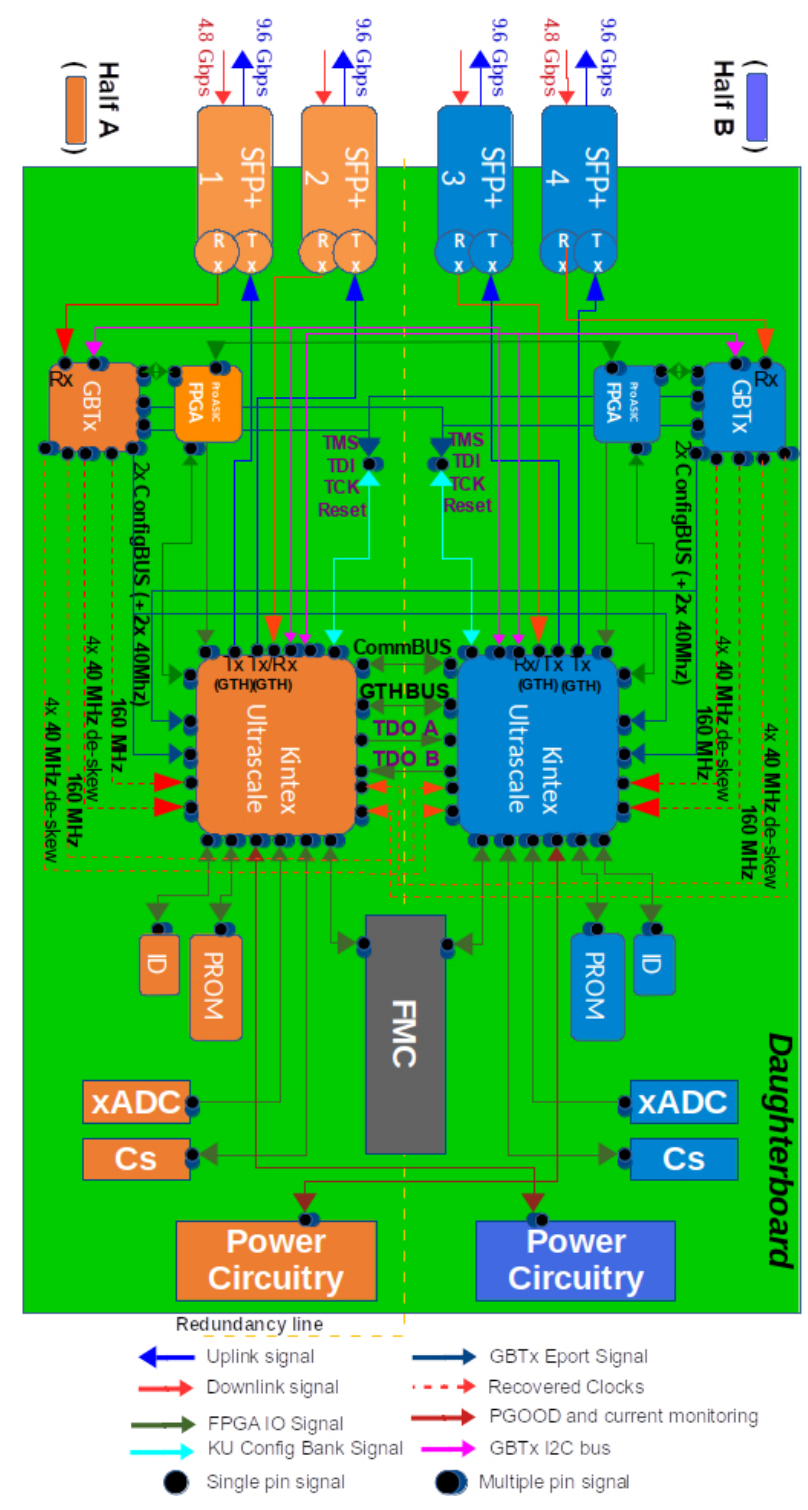

Fig. 9. DB revision 6 block diagram.

To evaluate their suitability for the new DB, we performed new test campaign at the CCB facility with a scanning proton beam at $226 \mathrm{MeV}$. For the tests, we used two Trenz TE0841 micromodules equipped with one Xilinx KU035 FPGA each, a Microsemi A3PE starter kit, and a Lattice ICEBLINK LP1K evaluation board. The FPGAs were exposed to a fluence of $1.2 \times 10^{11}$ protons $\times \mathrm{cm}^{-2}$ corresponding to ten years of HL-LHC running plus safety factors. The fluence was delivered over seven runs with two beam intensity settings. Some of the runs were paused due to beam interruptions and then continued until the full dose was delivered.

Fig. 8 shows the current evolution of the monitored currents for all the tested devices under the whole delivered dose. No SEL was detected for any of the devices. The test was divided into seven runs with runs 1 and 2 done with one Trenz micromodule and runs 3-7 done with the second one.
The beam intensity was raised after the third run to double the dose rate in runs 4-7.

To measure SEU rates through the JTAG chain, a firmware, including the Xilinx SEM, was loaded into the KU FPGAs. JTAG access to the Xilinx FPGAs was lost early in the runs, however, due to failure of a nearby buffering complex programmable logic device (CPLD) in the Trenz micromodules that was indirectly irradiated due to its proximity to the KU FPGA. Nevertheless, sufficient data were obtained to establish an SEU rate of around 166 SEUs per $10^{9}$ proton $/ \mathrm{cm}$ at $226 \mathrm{MeV}$, corresponding to approximately seven times the SEU rate of the KU+ at $58 \mathrm{MeV}$. This result was not unexpected and is considered well within what can be managed with SEM and TMR mitigation techniques.

It should be pointed out that the CPLD failures were the reason for changing the Trenz TE0841 boards between runs 2 and 3, as shown in Fig. 8. After replacing the failed CPLDs, full JTAG functionality was restored and the FPGAs were successfully tested. The ProASIC3 FPGA functionality became erratic at approximately $58.3 \mathrm{kRad}$ accounting for $75 \%$ of the total TID delivered. The behavior persisted after power cycling, indicating cumulative effects of the TID to the flash-based configuration memory. After a two-month annealing period, the firmware spontaneously returned to full functionality. However, the reconfiguration capabilities could not be recovered, presumably due to permanent damage to the charge pump mechanism on the FPGA. This outcome for the ProASIC3 agrees with results reported by Microsemi (Actel) in [10]. The FPGA of the Lattice ICEBLINK LP1K evaluation board functionality was affected after $10^{9}$ proton $/ \mathrm{cm}$. However, the functionalities were recovered after a reconfiguration of the device by means of a power cycle. Although both the Lattice and ProASIC3 FPGAs showed no SEL over the delivered fluence, we chose the ProASIC3 for the DB JTAG interface because the flash-based configuration is preferable for the DB6 design, and it has better development software support from the manufacturer. The reconfiguration capabilities of the ProASIC3 FPGAs are not of vital importance because the firmware is not expected to be changed after the DB production phase.

\section{DB REVISION 6}

From our experiences described above, the new DB design (DB revision 6 or DB6) aimed to eradicate SEL radiation issues detected in DB5 and incorporate a more robust power circuitry with overcurrent mitigation, a more robust timing design, and fewer single points of failure. At the same time, the new design should also maintain backward compatibility with previous interfaces.

In addition to ongoing qualification testing of the semiconductor components used, the DB6 design also replaces the large electrolytic capacitors used in the DB5 power circuitry with conductive polymer capacitors. This redesign choice was made on the basis of studies that report that conductive polymer capacitors show no performance deterioration from radiation up to $200 \mathrm{kRad}$ at $500 \mathrm{rad} / \mathrm{h}$ [7].

The DB6 features a power-up scheme that chains the dc-dc converters by means of the power-good output pins (PGOOD) 
and run-enable input pins (RUN) of the dc-dc converters, allowing to switch on each of the voltages in the order recommended by the manufacturers of the different chips used in the board. While we do not expect SEL in the DB6, a simple analog protection circuit has been added to protect against any form of overcurrent. The protection circuit is built from n-channel MOSFETS and includes a monostable circuit that is triggered when the analog output from one of the current monitoring amplifiers exceeds a safety threshold. This disables the entire $\mathrm{dc}-\mathrm{dc}$ converter chain on the affected DB half for a short period, allowing mitigation of any temporary latch-up like effect. All PGOOD signals and monitored currents are continuously sent to the off-detector system by the FPGA placed on the opposite half as part of detector control system (DCS) data. This extra layer of hardware control and monitoring contributes to minimize single points of failure and allows mitigation of any unexpected issues during nominal runs.

A block diagram for DB6 is shown in Fig. 9. The KU XCKU035 is used, which features GTH transceivers fully compatible with the GBTx ASIC clocks and with the LHC timing. Unlike DB5, the DB6 is capable of receiving four downlinks to allow loop-back testing of the high-speed links. "CommBUS" and "GTHBUS" are two new multipurpose independent communication buses between the FPGAs allowing signal monitoring, internal trigger commands, and data interchange. CommBUS is a slow bus using bidirectional double data rate buffers situated on the KU general-purpose I/O (GPIO) banks, while GTHBUS is a 9.6-Gb/s high-speed bus implemented with GTH transceivers.

As for DB5, the KU FPGAs read out the dual-gain digitized PMT data from the MB and format them into GBT-CRC protected words.

\section{CONCLUSION}

The DB is a read-out link and control board serving as a hub to interface the front-end electronics of a TileCal MD with the off-detector electronics. Unexpected observation of latch-up in the KU+ FPGAs on DB5 motivated us to migrate back to the previous KU family. The Ultrascale+ process was expected to have substantial improvement in radiation tolerance along with other advantages. The SEL sensitivity was discovered at a late stage of the DB design, after the production of the first prototypes. We observed SEL sensitivity at a late stage, but we found afterward that the issue had already been reported. However, it is clear that we were too early when adopting the Ultrascale + technology. Further improvements to the DB radiation tolerance include using conductive polymer capacitors with the $\mathrm{dc}-\mathrm{dc}$ regulators. The new DB board components have successfully passed SEL testing, while new TID, NIEL, and SEU tests will take place during the second half of 2021 to qualify the board as required by ATLAS radiation policies. The DB6 features new power circuitry with a controlled power-up sequence that is interfaced with a protection circuit that will power cycle the board half in the event of an overcurrent or a power failure. The design mitigates the previous problems related to the interface of the GBTx and the JTAG chain of the main FPGA by means of a ProASIC3 FPGA that controls, translates, and fans out the interface signals. Optimization of the ADC read-out modules and the complex DB clocking scheme was carried out to improve the firmware timing closure and assure good performance during physics runs. Dedicated phase configurable deskew clock paths were included to drive the charge injection calibration system independently of the ADC-read-out block. The DB6 functionalities are currently being evaluated, and a full set of radiation tests is planned for the near future.

\section{REFERENCES}

[1] The ATLAS Experiment at the CERN Large Hadron Collider, document JINST 3 S08003, ATLAS Collaboration 2008.

[2] Technical Design Report for the Phase-II Upgrade of the ATLAS Tile Calorimeter, document CERN-LHCC-2017-019 ATLAS-TDR-028, ATLAS collaboration, 2018.

[3] E. Valdes Santurio, S. Silverstein, and C. Bohm, "ATLAS tile calorimeter link daughterboard," in Proc. Topical Workshop Electron. Part. Phys. (TWEPP), vol. 343, 2018, p. 24, doi: 10.22323/1.343.0024.

[4] S. D. Lee, Single-Event Characterization of $16 \mathrm{~nm}$ FinFET Xilinx Ultrascale+ Devices with Heavy Ion and Neutron Irradation, document SAND2018-7621C, 2018, doi: 10.1109/NSREC.2018.8584313.

[5] D. M. Hiemstra, V. Kirischian, and J. Brelski, "Single event upset characterization of the Zynq UltraScale+ MPSoC using proton irradiation," in Proc. IEEE Radiat. Effects Data Workshop (REDW), Jul. 2017, pp. 1-4, doi: 10.1109/NSREC.2017.8115448.

[6] J. Karp, J. Michael Hart, P. Maillard, G. Hellings, and D. Linten, "Single-event latch-up: Increased sensitivity from planar to FinFET," IEEE Trans. Nucl. Sci., vol. 65, no. 1, pp. 217-222, Jan. 2018, doi: 10.1109/TNS.2017.2779831.

[7] M. Slovick. Capacitors for Spacecraft: Withstanding a Harsh Radiation Environment. Accessed: Aug. 15, 2021. [Online]. Available: https://www.ttiinc.com/content/ttiinc/en/resources/marketeye/categories/ passives/me-slovick-20160809.html

[8] J. Anderson et al., FELIX: A PCIe Based High-Throughput Approach for Interfacing Front-End and Trigger Electronics in the ATLAS Upgrade Framwework, document JINST 11 C12023, 2016, doi: 10.1088/17480221/11/12/c12023.

[9] P. Moreira, J. Christiansen, and K. Wyllie, GBTx Manual. Meyrin, Switzerland: CERN, 2021. [Online]. Available: https://espace.cern.ch/ GBT-Project/GBTX/Manuals/gbtxManual.pdf.

[10] ACTEL. (Apr. 2010). Radiation-Tolerant ProASIC3 FPGAs Radiation Effects. [Online]. Available: https://www.microsemi.com/documentportal/doc_view/131374-radiation-tolerant-proasic3-fpgas-radiationeffects-report 